\title{
Waiting list statistics. I: relation between admissions from waiting list and length of waiting list
}

\author{
M J GOLDACRE, A LEE, B DON
}

\begin{abstract}
The relation between changes in inpatient workload, measured as increases or decreases in the number of inpatients admitted from the waiting list, and the overall length of the waiting list was studied. Overall trends in admissions from the waiting list, the influence of seasonal patterns, and the impact of industrial action on admissions were also studied. The hypothesis was that when admissions from the waiting list increased the length of the waiting list would decrease and vice versa. No such simple relation was found. In fact, if anything, as the number of admissions from the waiting list increased so did the length of the waiting list.

This result could be due to inconsistencies in compiling waiting list data or to the use of waiting lists to improve organisational efficiency. It is also possible, and perhaps likely, that the ability to meet need in admitting patients to hospital influences patients and their doctors to translate previously unmet need into demand for hospital services.
\end{abstract}

\footnotetext{
Unit of Clinical Epidemiology, University of Oxford, Headington, Oxford OX3 7LF

M J GOLDACRE, BM, FFCM, director

A LEE, MB, FFARACS, World Health Organisation training fellow

Oxford Regional Health Authority, Headington, Oxford OX3 7LF

B DON, BSC, DPHIL, regional statistician

Correspondence to: Dr Goldacre.
}

\section{Introduction}

Long waiting lists for admission to hospital have been a feature of the National Health Service ever since its inception. The length of the waiting list-the number of patients on the waiting list for admission as inpatients at a particular time-is often used as an indicator of unmet need and is also sometimes interpreted as an indicator of inadequacy of services. Not so much detailed attention has been paid to the impact of changes in workload-that is, an increase or decrease in the number of patients admitted from the waiting list - on the overall length of the waiting list. We therefore investigated trends in the length of the waiting list, in the numbers of admissions from the waiting list, and the relation between the two.

\footnotetext{
Methods

We studied figures for general surgery, trauma and orthopaedic surgery, ear, nose, and throat surgery, gynaecology, ophthalmology, and plastic surgery in the Oxford region. These specialties account for about nine tenths of the patients on the waiting list in the region. Hospital Activity Analysis was used to obtain the numbers of patients admitted in each month from January 1974 to December 1983. The recording of "method of admission" in Hospital Activity Analysis allows the distinction to be made among waiting list admissions, booked admissions, accident admissions, emergency admissions, and transfers from other hospitals. During the period included in the study Hospital Activity Analysis in the Oxford region covered six of the present eight health districts (total covered resident population about 1.9 million). Routine statistics on the number of patients who are on the waiting list are available only quarterly: the numbers of patients on the waiting list at the end of each quarter in the same districts and specialties were obtained from the routine SBH 203 returns for March and September, from SH3 returns for December, and from mid-year returns from hospitals for June.

To make direct comparisons among data on admissions and data on length of the list, booked admissions were combined with waiting list admissions in
} 
Hospital Activity Analysis, as the SBH 203 and SH3 figures for the waiting list contain booked cases. The Department of Health and Social Security instructed health authorities to remove day cases from the figures on the length of the waiting list from the quarter ending September 1979, so these were also excluded from the admission data in our analysis from this period.

We obtained information about periods of industrial action within the region from the regional health authority's public relations department, supported by reports in the local and national press.
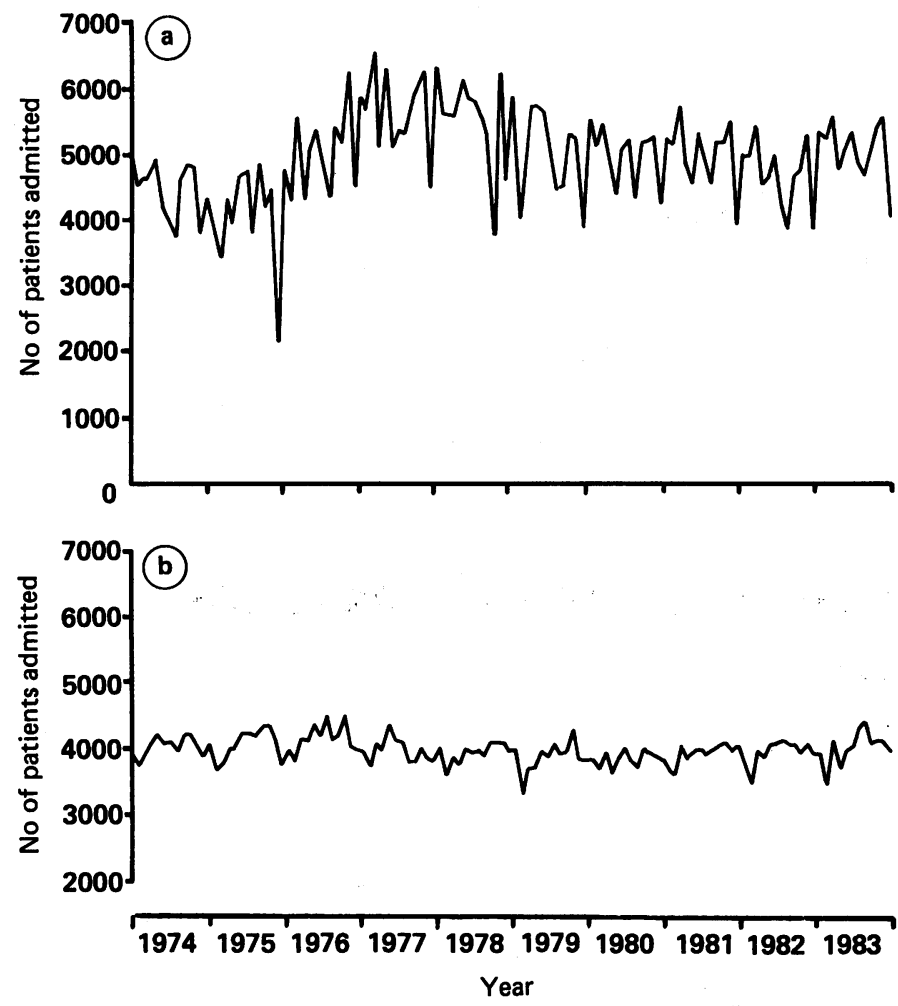

FIG 1-Number of admissions each month 1974-83 (a) from waiting list and (b) from sources other than waiting lists.

The data were analysed by the Box-Jenkins time series method, using the BMDP statistical analysis computer program P2T. ${ }^{1}$ The Box-Jenkins approach enables a series of models to be constructed to identify the contribution of each of several different effects on the time trends in a set of data. Each time series was "decomposed" by allowing for: dependence of the value at one point in time on the values taken at adjacent points (by moving average and autoregression models); seasonal variation; the effects of industrial action tested by fitting "external intervention" models; and random variation. The relations among various time series were studied by constructing transfer function models. ${ }^{1-3}$

\section{Results}

\section{MONTHLY ADMISSIONS TO HOSPITAL: SECULAR TRENDS}

Figure $1(a)$ shows that the number of patients admitted from the surgical waiting list each month between 1974 and 1983 fluctuated quite widely: the monthly median number of admissions from the waiting list was 5012, and the range was 2155-6509 (mean 4961, SD 703.4). The number of surgical patients admitted from other sources, which include the accident and emergency admissions, was much less variable. The monthly median was 3926 , and the range was 3226-4484 (mean 3919, SD 212 1 ). The number of admissions from the waiting list showed a general increase from 1974 to 1979, slightly declining thereafter. This trend was largely due to the omission of day cases. There were also some striking short term decreases in admissions, notably those that corresponded with periods of industrial action (see below).

Figure $1(b)$ shows that the number of people admitted to hospital from sources other than the waiting list was fairly constant. Overall there was a significant positive association between the change in the monthly number of patients admitted from the waiting list and the change in the number of patients admitted from other sources $(r=0.41, \mathrm{df}=117, \mathrm{p}<0.001)$. Admissions from the waiting list averaged $56 \%$ of all admissions.

\section{SEASONAL VARIATION}

The number of admissions from the waiting list tended to be particularly low in December and higher than average in November. These seasonal patterns were significant (autocorrelation coefficient at a lag of 12 months= 0.57 , SEM 0.16 after removal of trends).

The number of admissions from other sources was generally higher in the summer than in the winter, with February being particularly low. The increase in summer was mainly due to an increase in admissions of accidents. This seasonal variation was also significant (autocorrelation coefficient at lag of 12 months $=0 \cdot 55$, SEM $0 \cdot 12$ )

\section{INDUSTRIAL ACTION}

We examined whether periods of documented major industrial action in the region had affected admissions by fitting an intervention model that took account of the underlying secular trend and of seasonal effects. As an example of the interrelation between these factors the decline in admissions from the waiting list in December 1975 was attributable partly to the general downward trend from 1974 to 1975 , partly to the general seasonal December dip, and partly to a decline in admissions that was independent of either of these two factors. We considered in particular four months when industrial action was considered to have been widespread-namely, December 1975 , October 1978, February 1979, and August 1982. The shortfall in admissions from the waiting list in December 1975 that was "attributable" (at least in statistical terms) to industrial action was 1359 (SEM 368.8, $\mathrm{df}=102$, p<0.001). In October 1978 and February 1979 the average shortfall for the two months combined was 1694 admissions for each month (SEM 265.8, $\mathrm{df}=102, \mathrm{p}<0.001)$. The estimated shortfall in August 1982 was 392 admissions (SEM 366.8, NS). These figures are equivalent to about $6 \%, 6 \%$, and $1 \%$, respectively, of the length of the waiting list at the time. There was no significant decrease in the number of emergency admissions or accident admissions in these four months.

\section{LENGTH OF WAITING LIST: ABSOLUTE NUMBERS}

Figure 2 shows the number of patients who remained on the waiting list at the end of each quarter. For comparison, the numbers of patients admitted to hospital from the waiting list during each quarter are also shown. The number of people on the waiting list increased over the first five years covered by the study from about 20000 in 1974 to a peak of over 30000 in 1979. It dropped subsequently to around 25000 during the second five years. Most of the decline after 1979 is due to the omission of day cases from

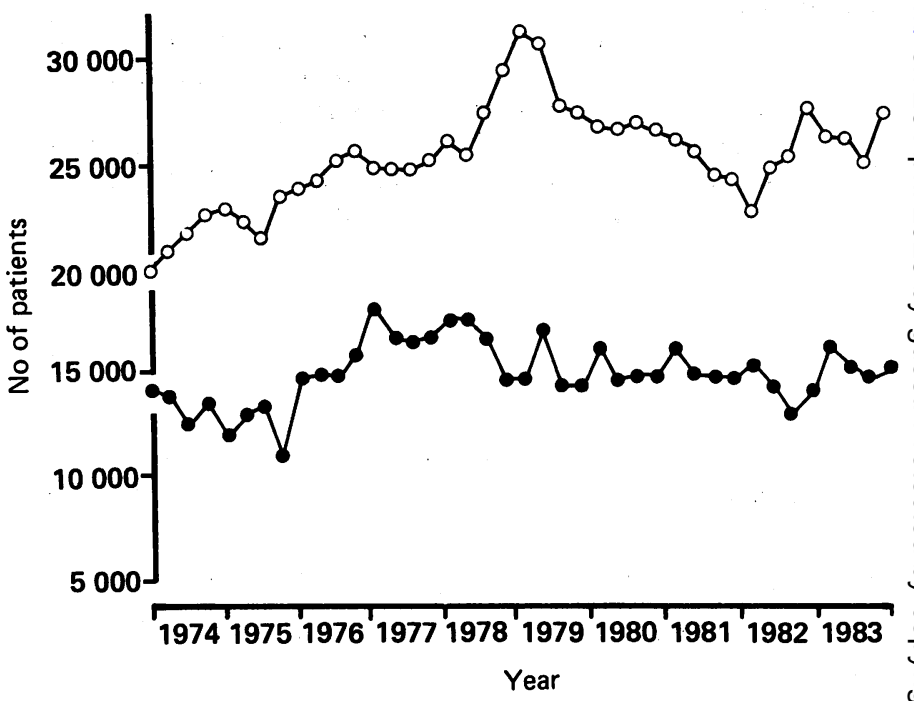

FIG 2-Number of patients on waiting list $(O)$ and admitted from waiting list $(O)$ for each quarter 1974-83. 
the statistics. Over the 10 years the median list length was 25668 (mean 25632 , range $20388-31239$, SD 2360). There was no significant seasonal quarterly pattern in the length of the waiting list.

Overall there was a significant positive correlation between the quarterly number of admissions from the waiting list and the length of the waiting list at the end of each quarter $(r=0.35, \mathrm{df}=38, \mathrm{p}<0.05)$. In other words, as the similarity of the secular trends in each variable in figure 2 suggests, an overall
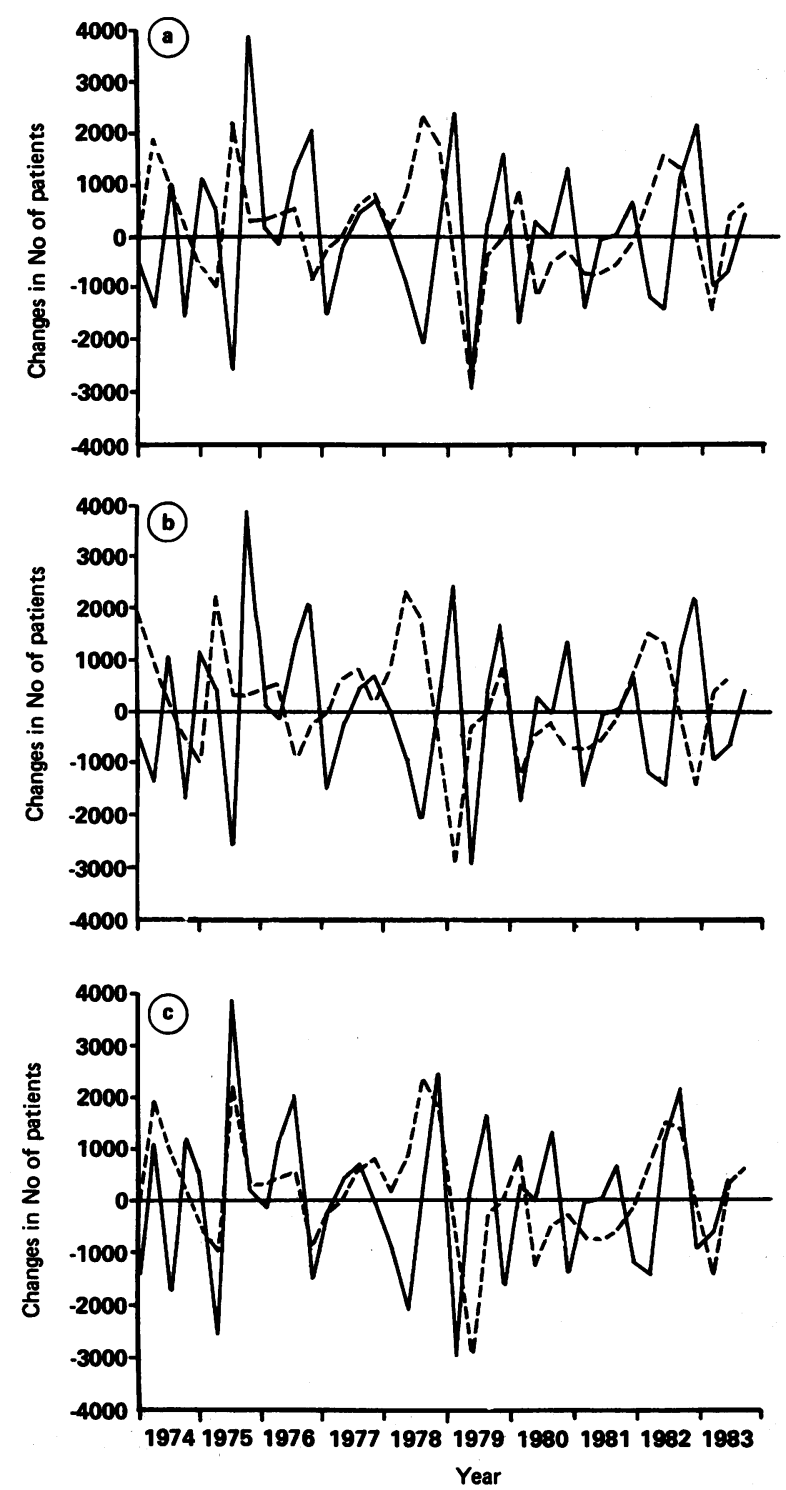

FIG 3 - Quarterly changes in number of patients on waiting list (---) and number of patients admitted from waiting list $(-)$. (a) No shift. $(b)$ One quarter backward shift in plot for number of patients on waiting list. (c) One quarter forward shift in plot for number of patients on waiting list.

increase in the number of admissions from the waiting list was paralleled by an overall increase in the number of people on the list. Similarly, as the number of admissions from the waiting list levelled out, so too did the number of people on the waiting list.

\section{LENGTH OF WAITING LIST: RATE OF CHANGE}

We investigated the relation between short term changes in the number of admissions from the waiting list during each quarter and changes in the length of the waiting list at the end of each quarter. There was no significant correlation between the quarterly differences in the length of the list and quarterly differences in admissions.

We then analysed the data more rigorously, using transfer function models. Firstly, to investigate the short term impact of changes in workload we tested whether an increase in the number of admissions from the waiting list during one quarter was associated with a corresponding reduction in the waiting list at the end of the quarter. This relation is shown by figure $3(a)$ and, as with the simple correlation reported above, we found no evidence of any short term association between activity levels and length of the waiting list.

Secondly, to investigate any delayed impact of changes in workload we tested whether an increase in the number of admissions from the waiting list in one quarter was associated with a corresponding reduction in the waiting list at the end of the following quarter, the quarter after that, and so on. Each pair of plots in figure $3(b)$ gives the change in the number of admissions during the quarter and the change in the length of the waiting list at the end of the following quarter. Again, the data did not support the hypothesis that a change in activity led to a change in the length of the waiting list.

Thirdly, we considered the possibility that an increase in the length of the waiting list in one quarter might lead to an increase in admissions from the waiting list of the following quarter, the quarter after that, and so on. Each pair of plots in figure 3(c) represents the change in the length of the waiting list at the end of each quarter and the change in the number of admissions from the waiting list during the following quarter. This association was significant-that is, a change in the length of the list at the end of each quarter was associated with a change in the same direction in the number of admissions from the waiting list in the following quarter (sample cross correlation $=0 \cdot 48$, SEM $0 \cdot 16, \mathrm{p}<0 \cdot 01$ ).

\section{Discussion}

We have shown that the monthly number of admissions from the waiting list varied substantially during the study. Identifiable causes of troughs in admissions were the regular seasonal decline and, more notably, the significant declines associated with industrial action in 1975 , in the winter of $1978-9$, and in 1982 . By contrast, the number of monthly admissions from other sources showed much less variation. This indicates that during the disputes the admission of acutely ill surgical patients was not compromised to any appreciable degree.

The main purpose of our study was to determine whether changes in the number of patients being treated from the waiting list were reflected in changes in the length of the waiting list. Our prior expectation was that when admissions from the waiting list increased the length of the waiting list would decrease and vice versa. We found no such obvious relation. The substantial increase in the number of patients on the surgical waiting list during the second part of the 1970s, far from being due to a general decline in the number of patients admitted to hospital for care, did in fact parallel an overall upward trend in the number of admissions from the waiting list. Indeed, it is interesting that national figures for a recent period have shown that the lengths of waiting lists have increased despite accelerated activity to admit patients from waiting lists during the preceding period. ${ }^{4}$ In our analyses of shorter term relations between the sets of data we did not find any statistical evidence that changes in the length of waiting lists followed changes in the number of patients treated from the lists.

This lack of relation runs counter to what might intuitively be expected and requires some attempt at explanation. Any short term decrease in the number of patients admitted from the waiting list must mean that those patients who are not admitted will remain on the waiting list and must mean further delays for those in need of care.

None the less, it is well recognised that figures on the length of waiting lists are difficult to interpret and that official statistics on the waiting lists often do not provide an accurate measure of the number of people awaiting care..$^{5-9}$ The data on the length of waiting lists may contain so many anomalies and inconsistencies that changes in these data are determined only to a minor degree by changes in the number of admissions from the waiting list.

A second possibility is that in some circumstances an increase in the length of waiting lists could reflect an increase in their use as a device for scheduling the admission of patients to hospital and thereby as a means of increasing organisational efficiency in the use of beds. In this circumstance an increase in the length of waiting lists might be expected as a means of actually increasing efficiency, to 
precede an increase in admissions from the waiting list. Another possible contributing factor is that most hospitals in the Oxford region have high figures for bed occupancy, high throughput figures, and short durations of stay. In these circumstances there is little spare capacity to accommodate the effects of even fairly minor, short term declines in admissions. Thus though workload may sharply decline below the typical monthly figures, it may not be possible for it to rise commensurately above it as simple compensation.

A final possibility is that the same factors that influence the numbers of inpatients treated may also influence the capacity to see outpatients awaiting surgery and therefore to enter them on to inpatient waiting lists. Thus with regard to short term fluctuations, as work declines in admitting patients from the waiting list so it may also decline in admitting new patients to the waiting list. Conversely, as the amount of inpatient activity increases so too may the amount of outpatient activity increase as the "gateway" to the waiting list. On a longer term basis it is also possible that the ability to meet demand acts as a positive influence for patients and their doctors to translate previously unmet needs into demand.

\section{References}

1 Liu LM. Box-Jenkins time series analysis. In: Dixon WJ, ed. BMDP statistical software. California: University of California Press, 1983.

2 Box GEP, Jenkins GM. Time series analysis: forecasting and control. 2nd ed. San Francisco: HoldenDay, 1976.

3 Helfenstein U. Box-Jenkins modelling of some viral infectious diseases. Stat Med 1986;5:37-47.

4 Hildrew P. Rise in hospital waiting lists upsets Fowler. Guardian 1986 Nov 25:2.

5 Williams B. The use and misuse of bed-occupancy and waiting list figures. Lancet 1968;i:1029-30. 6 Yates J. In-patient waiting list statistics: handle with care. Birmingham: Health Services Management Centre, University of Birmingham, 1978.

7 Lourie JA. Notes on an orthopaedic waiting list. Br $\mathcal{J}$ Clin Pract 1978;32:224-5.

8 Sanderson HF. What's in a waiting list? BrMed f 1982;285:1368-9.

9 Dalton KJ. Surgical waiting lists. Br Med f. 1984;289:495.

(Accepted 11 August 1987)

\title{
Logic in Medicine
}

\section{Doctors and witchdoctors: Which doctors are which?-II}

\author{
LARRY BRISKMAN
}

At the end of last week's discussion of inductivism and its criticism by Hume we found ourselves on the horns of a trilemma. Either we continue to insist that what characterises empirical science is its use of the inductive method, in which case we must find some way round Hume's argument (and other difficulties); or we must conclude that empirical science is indeed just a pseudoempirical superstition; or we must find an alternative solution to the problem of demarcation.

Most contemporary philosophers of science refuse to countenance the possibility that the theories of empirical science are simply pseudoempirical superstitions. In this they are, I think, quite right. For let us assume that we equate empirical science with pseudoempirical superstition. We shall then have to conclude that the attempt to bring the benefits of Western medicine to other parts of the world is simply a matter of cultural imperialism as Western medical science is really no better than are the various traditional, usually superstitious, medical practices of others. Yet no one takes such a possibility seriously for a moment-least of all the inhabitants of the so called "underdeveloped world," who are, in the main, crying out for these benefits. Thus the idea, which might be taken to follow from Hume's result, that the theories of empirical science are simply pseudoempirical superstitions, on a par with primitive myths, witchcraft, or magic, is hardly acceptable to anyone.

Rejecting this horn of the trilemma thus leaves us with only two options: either we find some way round Hume's argument (and the

Department of Philosophy, University of Edinburgh, Edinburgh LARRY BRISKMAN, MSC, PHD, lecturer in philosophy

This series is edited by Professor Calbert I Phillips. other problems facing traditional inductivism) or else we find some alternative, non-inductivist, solution to the problem of demarcation. The overwhelming majority of contemporary philosophers of science adopt the first tack. This is not to say that they still harbour the hope that Bacon's theory of induction can be made to work. Quite the contrary: most acknowledge the impossibility of a Baconian inductive method by which a theoretical understanding and explanation of phenomena may be obtained directly from these phenomena by a process of inference. Nevertheless, they continue to hold that empirical science is, when compared with myths, superstitions, and so on, a particularly secure and reliable body of knowledge as it is somehow well supported by empirical evidence. They hope either that an inductive "logic of confirmation" can be made to work without resorting to any pseudoempirical assumption or principle (perhaps because it requires no more than the logical or mathematical assumptions of the calculus of probability-for o example, Bayes's theorem) or that they can justify the use of induction pragmatically (as offering the best hope of achieving the aims of science).

\section{Popper's non-inductivist solution}

In opposition to all such attempts to salvage inductivism from the ravages of Hume's critique Sir Karl Popper ${ }^{1-5}$ has suggested an elegant non-inductive solution to the problem of demarcation-one that enables us to explain, despite Hume's arguments, why the theories of empirical science are to be preferred, from the point of view of truth, to those of witchcraft, scientology, and other pseudoempirical superstitions. According to Popper, what demarcates the theories of empirical science is not that they have been reached from observation by some special method of inference, or even that they are especially well supported by observation, but rather that they are open to observational and empirical criticism 